9 Draft Manuscript: Pattern formation in micromass culture micromass culture.

Rebecca A Rolfe*1 ${ }^{1}$, Claire Shea*1 and Paula Murphy ${ }^{1}$ of Dublin, Ireland

*Authors contributed equally to the work Dublin, Dublin 2, Ireland.

$$
\text { Phone: }+3531896-3580
$$

Email: paula.murphy@tcd.ie

Draft for re-submission following first review to

Cell and Tissue Research 2

\title{
Geometric analysis of chondrogenic self-organisation of embryonic limb bud cells in
}

${ }^{1}$ Department of Zoology, School of Natural Sciences, Trinity College Dublin, The University

Correspondence: Department of Zoology, School of Natural Sciences, Trinity College

Keywords: Chondrogenesis, micromass culture, self-organisation, pattern formation, ArcGIS 


\section{Abstract}

36 Spatial and temporal control of chondrogenesis generates precise, species-specific patterns of skeletal structures in the developing vertebrate limb. The pattern-template is laid down when mesenchymal cells at the core of the early limb-bud condense and undergo chondrogenic differentiation. Although the mechanisms involved in organising such complex patterns are not

40 fully understood, the interplay between BMP and Wnt signal

41 ing pathways is fundamental. Primary embryonic limb bud cells grown under high-density 42 micromass culture conditions spontaneously create a simple cartilage nodule pattern, presenting a model to investigate pattern generation. We describe a novel analytical approach to quantify geometric properties and spatial relationships between chondrogenic condensations, utilizing the micromass model. We follow the emergence of pattern in live cultures with nodules forming at regular distances, growing and changing shape over time. Gene expression profiling supports rapid chondrogenesis and transition to hypertrophy, mimicking the process of endochondral ossification within the limb bud. Manipulating the signaling environment through addition of BMP or Wnt ligands, as well as the BMP pathway antagonist Noggin, altered the differentiation profile and nodule pattern. BMP2 addition increased chondrogenesis while WNT3A or Noggin had the opposite effect, but with distinct pattern outcomes. Titrating these pro- and anti-chondrogenic factors and examining the resulting patterns, supports the hypothesis that regularly spaced cartilage nodules formed by primary limb-bud cells in micromass culture is influenced by the balance of Wnt and BMP signaling under a Turing-like mechanism. This study demonstrates an approach for investigating the mechanisms governing chondrogenic spatial organization using simple micromass culture. 


\section{Introduction}

Micromass culture is a well-established approach for assaying chondrogenic potential, where cells are plated in a simple high-density, microdrop culture, that can induce cellular aggregation and chondrogenic differentiation, mimicking the process of mesenchymal condensation during skeletogenesis (reviewed in Bobick et al., 2009). A major advantage of this system is its simplicity, where chondrogenesis can be monitored temporally. It is a highly adaptable model that allows for testing of a variety of conditions including the application of soluble factors 77 (Bobick et al., 2014; Francis-West et al., 1999; Mello and Tuan, 2006) and the effects of mechanical stimulation (Juhasz et al., 2014; Klumpers et al., 2015; Saha et al., 2017). A striking aspect of micromass cultures made from primary embryonic limb bud cells is the generation of a self-organised spatial pattern of chondrogenic nodules (Butterfield et al., 2017; Christley et al., 2007; Mello and Tuan, 1999; Raspopovic et al., 2014; Saha et al., 2017). This innate property is also demonstrated in the embryonically-derived cell line ATDC5 (derived from mouse embryonic teratocarcinoma) (Seemann et al., 2005); however, the cell line C3H10T12 (derived from mouse embryonic sarcoma) fails to form distinct nodules, yet expresses chondrogenic marker genes (Takacs et al., 2013). Thus, the capacity to spontaneously form regular, spatially-organised patterns of cartilage nodules is preserved in one of the embryonically derived cell lines and not the other. Although little is known about the mechanisms that underpin this self-assembly process, the micromass culture system provides a convenient opportunity for further investigation.

In the developing limb, spatial and temporal control of chondrogenesis and condensation of chondrogenic cells generates precise, species-specific patterns of limb skeletal structures. The majority of cells within mesenchymal cell condensations at the core of the developing limb bud differentiate into transient cartilage (marked by activation of Sox 9 and Col2al genes), later undergoing hypertrophy (marked by activation of Ihh and CollOal genes), followed by ossification to form the early bones (reviewed in Long and Ornitz, 2013). Cells at the joint interface however do not progress to hypertrophy but rather form the stable, permanent, articular cartilage of the future joint. The precise molecular mechanisms and physical cues that define these distinct, spatially appropriate differentiation programs, have not been fully elucidated. Recent work examining the effect of biophysical stimulation has shown that canonical Wnt signaling and BMP signaling are two major pathways involved in the distinctive differentiation of these two cartilage types within the rudiment (Ray et al., 2015; Rolfe et al., 2018; Singh et al., 2018). 
It is well-recognised that morphogen gradients are responsible for establishing proximo-distal and antero-posterior axes in the limb bud, and models incorporating Turing reaction-diffusion mechanisms have been proposed to explain the robust formation of skeletal elements in the limb skeleton (reviewed in Delgado and Torres, 2016; Glimm et al., 2020). Specifically, the interaction of BMP and Wnt signals has been proposed to regulate Sox9 expression and the formation of regularly-spaced digits within the digital plate in a Turing-like reaction-diffusion mechanism (Raspopovic et al., 2014). In this model, BMP and canonical Wnt signaling act as pro- and anti-chondrogenic factors to generate characteristic patterns within the digital plate, proposed to be reflected in the pattern generated in micromass cultures. This study further showed that both Sox9-positive (pre-cartilage) and -negative (non-cartilage) cells derived from embryonic limb buds are capable of establishing nodule patterns in micromass culture, demonstrating that the formation of cellular condensations is not based on pre-determined cell fate but on whole-culture dynamics, which could be influenced by concentration/diffusion of signaling molecules and mechanical characteristics within the culture.

A robust method to quantitatively describe and assess condensation formation in micromass culture would therefore provide a means of examining proposed mechanisms that underlie pattern generation in vitro, allowing more comprehensive comparison of the patterns formed under different conditions. Some studies have employed sophisticated modelling approaches to investigate chondrogenic pattern formation in micromass culture (Christley et al., 2007; Miura and Shiota, 2000a, b), others have measured periodicity and the shape and size of individual nodules (Butterfield et al., 2017; Duke et al., 1998) and more recently intercondensation distances (Bhat et al., 2019). In this study, we develop novel methods to systematically quantify the spatial pattern of chondrogenic condensations that form from embryonic limb bud-derived cells in an experimental setting. We examine the self-organising pattern as it emerges and changes over time and how it responds to changes in the signaling environment by addition of soluble factors. We demonstrate that modulation by treatment with BMP2 or WNT3A recombinant proteins results in opposing effects on the self-organisation pattern, with BMP resulting in more nodules that are closer together, while WNT3A reduces condensation formation. Furthermore, we demonstrate that titration of these pro- and antichondrogenic signaling molecules adjusts the balance of condensation formation. This study expands our knowledge on the balance of signaling molecules involved and also presents an experimental method for quantifying biological spatial patterns in vitro.

\section{Materials and Methods}


CD1 mice housed in the Bioresources Unit Trinity College Dublin were mated over-night. The morning of detection of vaginal plugs was designated as Embryonic day (E) 0.5 with collection of embryos on E10.5, E11.5 and E12.5 according to the regulations of the HPRA (Health Products Regulatory Authority), Ireland, by a licensed individual. Micromass cultures were set up as detailed in Saha et al. (2017), with minor adjustments. Micromass drops (15 $\mu 1)$ were plated at a density of 1-2 $\times 10^{7}$ cells per ml either directly on tissue-culture grade treated plastic dishes (Thermo Fisher/Nunc 176740 or 142485), or on $10 \mathrm{~mm}$ glass coverslips (Invitrogen 12658116) pre-treated with $0.015 \mathrm{M}$ poly-D-ornithine (Sigma). Drop cultures adhered in an incubator $\left(37^{\circ} \mathrm{C}\right.$ and $\left.5 \% \mathrm{CO}_{2}\right)$ before being flooding with $400 \mu \mathrm{l}$ of growth medium (Dulbecco's F-12 Ham's medium [DMEM/F12] [Sigma] plus 2 mM L-Glutamine [Life Technologies], 100 units/ml Penicillin/Streptomycin [Life Technologies], $50 \mu \mathrm{g} / \mathrm{ml}$ Ascorbic acid [Sigma] and 10\% Fetal bovine serum [FBS; Sigma]) alone or supplemented with either human WNT3A (R\&D Systems 5036-WN), human BMP2 (R\&D Systems 355-BM) or human Noggin (R\&D Systems 719-NG) for final concentrations between 10-200ng/ml. The medium was changed on alternate days by removing half the well volume and replacing it with an equal volume of fresh medium. Cultures were harvested every 12 hours or every 48hrs until 144hrs.

\section{Alcian Blue staining}

Micromass cultures were washed in Phosphate Buffered Saline (PBS), fixed in 4\% Paraformaldehyde (PFA) in PBS for 30 mins, rinsed twice in PBS and stained with 1\% Alcian Blue (8GX, Sigma A5268) in 0.1 M HCL for 30 mins at room temperature. Cultures were washed and mounted on glass slides or stored in PBS. Slides were photographed using an Olympus SZX12 microscope; images of whole micromass cultures were taken on an Olympus dissecting microscope.

\section{Image analysis and nodule geometries}

Images of Alcian Blue-stained micromass cultures were processed using ImageJ (Version 1.51j8; National Institutes of Health, USA). Original files (Fig 1a) were cropped to define the region of interest (ROI) to a circle of $5 \mathrm{~mm}$ diameter which was centred over the micromass (Fig 1b). Images were 'cleaned' by encircling debris/ artefacts (not nodules) with the Freehand tool and filling the enclosed space with white (Fig 1c, red arrow). Cleaned images were split into component colour 'channels' (Fig 1d-d'), the green channel was determined to have the best signal-to-noise ratio and so was used for further analysis (Fig 1d'). Images were converted 
171 to an 8-bit black and white image by setting a minimum threshold, using the auto-threshold function as a guideline. This converted every pixel above the threshold in the greyscale green channel image to black, and every pixel below the threshold to white (Fig 1e). Treatment groups within an experiment had the same threshold value applied, but the appropriate threshold values were determined independently for each experiment, due to unavoidable variability in staining contrast or intensity, and in image capture. Any background noise was removed (Fig 1e, red arrowhead) and converged nodules were separated using the Despeckle and Watershed functions (Fig 1f, green arrowhead). At this stage, images were further cropped to a circular ROI with a $2-\mathrm{mm}$ diameter, centred within the larger $5 \mathrm{~mm}$ region of interest. Both the $5 \mathrm{~mm}$ and $2 \mathrm{~mm}$ images were used for analysis.

Nodules were counted and measured using the Analyse Particles function (Fig 1g), with a minimum particle size of $0.005 \mu \mathrm{m}^{2}$. The total nodule count was done using the 5 -mm ROI. Manual counts of nodule number were compared to automated counts, to ensure accuracy of the method; no difference was found between manual and automated counts (data not shown). Other parameters, including the size of each detected nodule (in $\mu \mathrm{m}^{2}$ ), the area covered by nodules $(\%)$ and shape descriptors such as circularity and solidity were based on the 2-mm ROI. Nodules touching the edge of the circular frame were excluded to prevent inaccurate size measurements. Exclusion of nodules at the edges could affect the measurements of nodule spacing and proximity slightly, but this was tested, and the difference was determined not to be significant. Automation of the procedure was performed by establishing command macros within ImageJ (Supplemental material 1). At earlier time points (i.e. $<48 \mathrm{hrs}$ ), cell condensations are very faint in appearance, even after Alcian Blue staining, and automated processing ensures that nodules are counted accurately by setting a threshold level. Automated watershedding, as described above, separates detected objects which overlap. When counting manually, these individual nodules were sometimes not easily distinguishable, especially at later time points (e.g. 144hrs), when nodules had grown and their edges appeared merged. Therefore, watershedding effectively separated nodules in an unbiased manner.

The Analyse Particles function generated shape measures of circularity and solidity for each detected nodule. Circularity is the measure of how close to a perfect circle a given shape is, as calculated by $4 \pi$ times the shape area divided by the shape perimeter squared. A perfect circle has a circularity value of 1.0; as the shape becomes more elongated (ellipsoid), its circularity value decreases (Fig 1h). Solidity is an indicator of the convexity, or "smoothness", of an object, as calculated by shape area divided by the convex hull area (Fig 1i). The convex area is the area of the smallest ellipse that can be drawn around the object, completely enclosing it. A 

234

circle or ellipse has a solidity value of 1.0; if the shape has more concave features (is "rougher"), the solidity value decreases.

To quantify nodule to nodule spatial relationships, the spacing and proximity of nodules were calculated (Fig 1j-k). To measure spacing, the distance between a given nodule's centre point and the nearest centre point of another nodule was calculated for each nodule in each micromass (Fig 1j, red dots). Image J Analyse Particles function generated $\mathrm{x}$ and y co-ordinates of the centre point of each detected nodule, which were analysed using the spatial point pattern analysis package "Spatstat" in RStudio (v.1.1.463) (Fig 1g), To measure proximity a method was devised, based on Geographical Information Systems (GIS) analysis, where the distance between a point on the perimeter of a given nodule and the closest point on the perimeter of a distinct neighbouring nodule was calculated (Fig 1k, green dots) using ArcGIS software and Python programming (v.3.7.2) (Fig 1k). Details of the script used for nodule spacing (nearest neighbour distances - centre-points) and nodule proximity (nearest neighbour distances edges) are specified in detail in supplementary material 2.

A minimum of 3 biological replicates were analysed in 3 independent experiments for cultures up to and including $48 \mathrm{hrs}$, and a minimum of 5 independent biological replicates in independent experiments for 48 hrs, 96 hrs and $144 \mathrm{hr}$ cultures. Each experiment consisted of 2-6 replicates in each group, that were processed for Alcian Blue staining and pattern analysis. SPSS statistics (IBM ${ }^{\circledR}$, v.26) was used for statistical analysis. In order to assess significance over time and between groups at each timepoint, one-way ANOVA was performed, followed by Tukey's post hoc test with a 95\% confidence interval. P values of $\leq 0.05$ were considered significant.

RNA extraction, cDNA synthesis, and real time quantitative gene expression analysis by Reverse Transcription-Polymerase Chain Reaction (RT-PCR)

230 For RNA extraction, individual micromass cultures were treated with $100 \mu 1$ TRIzol reagent for 2312 mins. Following mechanical homogenisation of the cell-Trizol solution with a pipette tip, 232 multiple micromass cultures (2-4 per growth condition) were pooled to increase RNA quantity 233 for analysis. Day 0 control RNA was obtained from 10-30 $\mu 1$ of cell suspension added to TRIzol on the day of cell harvest. All samples were stored at $-80^{\circ} \mathrm{C}$ until further processing. RNA was extracted with chloroform and purified with a TRIzol Purelink kit (Invitrogen) or an RNA miniprep kit (Qiagen) following manufacturers' instructions. Typically, RNA was resuspended in $30 \mu \mathrm{l}$ of nuclease-free water and quantified using the Qubit 2.0 Quantitation System (Invitrogen). 
239 Primers were designed using Primer Express Software ${ }^{\circledR}$, version 3.0 under default settings for $\operatorname{TaqMan}^{\circledR}$ quantification and purchased through Sigma (Sigma-Aldrich, Ireland), as listed in Table 1; Gapdh was used as the normaliser transcript. mRNA was reverse transcribed using a standard quantity of total RNA (100 ng) and High Capacity cDNA Reverse Transcription Kit (Applied Biosystems ${ }^{\mathrm{TM}}$ : 4368814), diluted 1:5 with RNase free water. Real-time PCR quantification was performed using an ABI 7500 as described in Saha et al. (2017). Three technical replicates were processed within each qPCR assay. A minimum of 3 independent experimental replicates for all cultures up to and including $48 \mathrm{hrs}$, and a minimum of 5 independent experimental replicates for Growth medium conditions and 2-5 independent experimental replicates for Bmp2 and WNT3A protein addition for $48 \mathrm{hr}, 96 \mathrm{hr}$ and $144 \mathrm{hr}$ cultures were combined for analysis.

\section{Results}

\section{Chondrogenesis and establishment of an organised pattern of cell condensations occurs} rapidly in micromass cultures

Primary limb bud cells obtained from E11.5 embryos grown in high density micromass cultures in Growth Medium (GM), show the first appearance of visible cell condensations by 24 hours (hrs) (Fig 2a). Tracking these condensations over time confirmed that they correspond to the site of future chondrogenic nodules, first clearly identifiable through Alcian Blue staining by $48 \mathrm{hrs}$, becoming more distinct at 96 and $144 \mathrm{hrs}$ (Fig 2a \& b). Molecular profiling of chondrogenesis using qRT-PCR to monitor expression of marker genes confirmed early chondrogenic differentiation (Supplementary Fig 1), in line with previous studies (Saha et al., 2017). The chondrogenic marker Col2al was significantly upregulated by 96 hrs $(14.7 \pm 4.4$ fold; $26.5 \pm 7$ Fold at $144 \mathrm{hrs}$ ), with an earlier increasing trend in mean expression values from $36 \mathrm{hrs}$ (compared to E11.5 limb bud cells at time 0 [prepared for plating]). Expression of Col10a1, a marker of hypertrophy, increased significantly at $144 \mathrm{hrs}(15.5 \pm 7.5$ fold, $\mathrm{p}=0.034)$. These data represent 5-6 independent experiments with high levels of variation between experiments, particularly at $144 \mathrm{hrs}$. Although mean expression of Sox9 (early chondrogenic marker) and Ihh (pre-hypertrophic marker) increased over time, in the context of the large variation between experiments, neither reached significance at the $95 \%$ confidence level. Elevation of the mean expression level of Ihh, at $36 \mathrm{hrs}(8.1 \pm 1.6$ fold, $\mathrm{p}=0.07)$ aligns with previously reported data for a transient up-regulation already at day 1 of culture (Saha et al., 
2017), and overall the trends align with rapid chondrogenic differentiation and progression to hypertrophy (Supplementary Fig. 1).

The visible pattern of chondrogenesis was considerably different with cells obtained from earlier (E10.5) or later (E12.5) limbs buds (Supplementary Fig 2), corresponding to previous reports in the chick limb (Ahrens et al., 1977): Using E10.5 limb bud cells, nodules were significantly larger, while nodules were significantly smaller from E12.5 limb bud cells. While there was a higher average number of nodules formed from E11.5 limb buds, this was variable and not significant (Supplementary Fig 2).

In order to describe the pattern of chondrogenic nodules in a systematic and quantitative way, we developed an automated, high-throughput process to capture features of the pattern: number of nodules, nodule size, area covered by nodules, circularity, solidity, spacing between nodule centres and proximity of nodule edges (Fig 1; Fig 2c-i). The number of nodules increased dramatically from $48 \mathrm{hrs}$ to $96 \mathrm{hrs}(6 \pm 1$ [48 hrs] to $240.9 \pm 19.4$ [96 hrs], $\mathrm{p} \leq 0.001)$, with no further increase to $144 \mathrm{hrs}$ (Fig 2c). The average \% area covered by nodules (in a $2 \mathrm{~mm}$ ROI) significantly increased at $96 \mathrm{hrs}$, further increasing by $144 \mathrm{hrs}(12.5 \pm 1.9 \%$ and $20.2 \pm 1.4 \%$, respectively $\mathrm{p} \leq 0.001 ;$ Fig $2 \mathrm{~d}$ ). This increase in area coverage corresponds to increase in nodule size, particularly between $96 \mathrm{hrs}$ and $144 \mathrm{hrs}$ (Fig 2d, e). These descriptors reflect observed nodule formation (Fig 2a, b) with no new nodules forming but nodules expanding in size between $96 \mathrm{hrs}$ and $144 \mathrm{hrs}$. Note that as adjacent nodules increase in size some form continuous lines of Alcian Blue staining (Fig 2a').

Individual nodule shape was analysed to monitor the characteristics of nodule growth using circularity and solidity parameters. Circularity is a measure of how close to a perfect circle a given shape is (value of 1.0); the lower the value, the less circular the shape. The average nodule circularity score decreased from $48 \mathrm{hrs}(0.98 \pm 0.01)$ to $96 \mathrm{hrs}(0.82 \pm 0.01)$ with nodules becoming less circular as they mature and enlarge (Fig $2 \mathrm{~g}$ ). Solidity is an indicator of how smooth the outline is with lower values reflecting a "rougher" shape. Nodules became "rougher" during the period of nodule formation (between 2 and 4 days) but once all nodules are formed, they become smoother (between 96 and 144 hrs) (Fig 2h).

300 For nodule-to-nodule relationships, while there is a dramatic drop in distances between $48 \mathrm{hrs}$ 301 and later timepoints, between 96 and $144 \mathrm{hrs}$ there is no change in the distance between nodule 302 centres (which corresponds to no new nodules being formed) and a decreasing trend in distance 303 between nodule edges (although not significant, this reflets the increase in nodule size). 304 Strikingly, the large variance in distance measurements at $48 \mathrm{hrs}$ reduces at later time points 
indicating that the pattern becomes more regular as it establishes, with constant distance between nodule centre points and edges.

\section{Manipulation of the signaling environment alters the pattern of chondrogenesis with} BMP and Wnt signaling pathways acting as pro and anti-chondrogenic factors.

We investigated the effect of altering the BMP and Wnt signaling environment on the establishment of the self-organised pattern of chondrogenic nodules in micromass cultures compared to growth medium. Upon addition of 100ng/ml BMP2 (bone morphogenetic protein 2) protein, clearly identifiable Alcian Blue-positive chondrogenic nodules were observed by $31448 \mathrm{hrs}$, similar to GM conditions (Fig 3a,b). Treatment with 200ng/ml WNT3A protein resulted 315 in a dramatic reduction in the formation of Alcian Blue-positive nodules across the entire 316 timeframe examined (Fig 3c), with replicate experiments showing no or very few stained 317 nodules at 96 and 144 hrs (Supplementary Fig 3).

318 Geometric analysis of the nodule pattern showed an increase in the average number of nodules 319 over time with added BMP2, with a temporal profile similar to GM conditions, but with an 320 increase in the number of nodules formed under BMP2 treatment at $144 \mathrm{hrs}(310.3 \pm 23.5)$ 321 compared to GM alone $(220 \pm 20.1, p \leq 0.01)$ (Fig 3d). There was also a significant increase in 322 the area covered by nodules (Fig 3f), while the average size of nodules at $144 \mathrm{hrs}$ did not 323 increase (Fig 3e). The distance between nodule centre points and nodule edges was 324 significantly reduced at 144 hrs with BMP2 treatment compared to GM (Fig 3h, i). Thus, at $325144 \mathrm{hrs}, \mathrm{BMP} 2$ treatment resulted in a larger number of distinct nodules, spaced closer together, 326 which therefore cover a larger area, but are not individually larger in size.

327 Contrastingly, with WNT3A addition there was a reduction in the number of nodules, nodule 328 size and therefore area coverage (Fig 3d, e, f). The shape of the WNT3A treated nodules present 329 at $96 \mathrm{hrs}$ and $144 \mathrm{hrs}$ were more circular than those in the GM or BMP2 groups (Fig 3g). A 330 striking effect of WNT3A addition was the irregular spacing of the few nodules that formed 331 (Supplementary Fig 3 represents the variability seen at $144 \mathrm{hrs}$ with 200ng/ml WNT3A 332 treatment across six experiments); therefore, due to enormous variation in the patterns 333 produced in the +WNT3A cultures, inter-nodule relationship data are presented for GM and $334+$ BMP2 cultures only. (The variation in nodule spacing seen on the addition of WNT3A is explored further in the next section). Taken together, these data reveal that while addition of BMP2 increases nodule formation over $144 \mathrm{hrs}$, resulting in more nodules with centres and edges closer together, activation of canonical Wnt signalling significantly reduces the appearance of nodules and the pattern is more irregular. Analysis of these geometric descriptors 
339 demonstrates that introduction of signalling molecules alters the chondrogeneic profile and pattern of cartilage condensations, with BMP and canonical-Wnt signalling acting as pro and anti-chondrogenic factors.

qRT-PCR analysis showed that addition of BMP2 results in a profile of chondrogenic and hypertrophic marker expression over time similar to GM, for example, increase in expression of Col2al reaching significance at $96 \mathrm{hrs}(14.9 \pm 5.9$ fold, $\mathrm{p}=0.002)$ and $144 \mathrm{hrs}(15.1 \pm 4.1$ fold, $\mathrm{p}=0.001$ ) (Fig 3j; Supplementary Fig 4). Sox9 expression levels showed a significant increase at $48 \mathrm{hrs}(2.6 \pm 0.4$ fold, $\mathrm{p}=0.02)$, whereas GM produced a more steady increasing trend over time (Supplementary Fig 4). Higher overall expression levels were observed for the pre-hypertrophic chondrocyte marker Ihh, in the presence of BMP2 with a significant increase compared to time 0 at 36 hrs (22.1 \pm 5.2 fold, p=0.04; Supplementary Fig 4) and significant increase compared to GM at $24 \mathrm{hrs}(20.1 \pm 7$ fold, $\mathrm{p}=0.018$; Fig $3 \mathrm{k})$. Expression of the hypertrophic maturation marker Col10al also demonstrated a similar profile to GM with increase over time, although with consistently higher mean levels (e.g., at $36 \mathrm{hrs} 13.4 \pm 5.4$ fold compared to $8.19 \pm 5.9$ fold; at $48 \mathrm{hrs}, 11.2 \pm 3.6$ fold compared to $4.5 \pm 2$ ) (Supplementary Fig 4) consistent with increased Ihh expression and an earlier shift toward a hypertrophic chondrogenic phenotype.

With addition of WNT3A the gene expression profile supports the nodule pattern analysis with consistently low levels of chondrogenic markers Sox9 and Col2a1; Col2a1 is already significantly downregulated at $12 \mathrm{hrs}$ compared to time 0 (Fig 3j and Supplementary Fig 4) and both Col2a1 and Sox9 are downregulated compared to GM and/or BMP2 conditions across the time profile. Markers of hypertrophic maturation (Ihh and Col10a1) in the presence of WNT3A show large variability across experiments ( $\mathrm{n}=3$ )-and, surprisingly, show transient up-regulation at 12 hrs (Ihh; Fig 3k) and 24 hrs (Col10a1; Supplementary Fig 4) compared to time 0. There were no significant differences compared to GM conditions for markers of hypertrophy, although the large variability should be noted.

These results, taken together, suggest that the qualitative and quantitative profile of chondrogenesis in GM, with rapid chondrogenic differentiation once cells are plated in high density micromass cultures, is enhanced with addition of BMP signaling ligand. In contrast, activation of Wnt signaling prevents chondrogenic differentiation.

Titration of the effects of BMP and Wnt: Adjustment of the equilibrium of signaling molecules alters the chondrogenic pattern in micromass culture. 
372 In order to further investigate the opposing effects of BMP and Wnt on the chondrogenic pattern in micromass culture, we titrated the concentration of added BMP2 and WNT3A proteins, examining the resulting pattern of nodules after $144 \mathrm{hrs}$. For comparison, to further explore the mechanism, we additionally investigated the effect of adding the BMP pathway antagonist, Noggin (NOG). Fig 4a shows nodules formed as a proportion of the number of nodules formed in GM when the concentration of each additive is varied between $50-200 \mathrm{ng} / \mathrm{ml}$ for nodule size, area, spacing and proximity. Supplementary Table 1 shows the direction of change and level of significance for all parameters and comparison of all treatments with GM (Supplementary Table 1a), as well as intertreatment comparisons (Supplementary Table 1b). It is clear that as the concentration of added BMP2 increases the number of nodules increases, the size of individual nodules does not, while the spacing between nodules decreases. With addition of the lowest concentration of BMP2 $(10 \mathrm{ng} / \mathrm{ml})$, there is already a significant increase in nodule number and significant decrease in spacing. The dose effect is not linear.

The addition of WNT3A and Noggin both lead to progressively fewer nodules, reduced nodule size and increase in the spacing between nodules as increasing amounts of protein are added. Addition of the lowest doses of WNT3A $(50 \mathrm{ng} / \mathrm{ml})$ and Noggin $(10 \mathrm{ng} / \mathrm{ml})$ had little effect on any parameter while further increasing dosage of either showed reductions in nodule number and size and increase in spacing.

To get an overview comparison of the effects, Fig 4e shows trend traces for the average values for several parameters across dosages. The opposing effects of both WNT3A and NOG compared to BMP2 are striking across parameters. Comparing WNT3A and NOG effects however we see that while they both reduce chondrogenesis, the effects are distinct. Decreases in nodule number, size and coverage are closer to linear for NOG than WNT3A (Fig 4e), for example as the NOG concentration was reduced there was a linear reduction in the extent of the difference in number, size and area coverage, with the intermediate concentration $(50 \mathrm{ng} / \mathrm{ml})$ still showing significant differences to GM (Fig 4b-d, Supplementary Table 1a). While circularity increases with both additives, the effect is more dramatic with WNT3A. More strikingly, while both factors lead to increase in the spacing, the regular spacing between nodules that is characteristic of micromass patterns ismore dramatically lost with the addition of WNT3A; this is reflected in the very large variance in spacing and proximity measurements upon addition of 100ng and 200ng/ml WNT3A (Fig 4c; Supplementary Fig 5), where regular spacing is lost. To further demonstrate the increased variability in nodule spacing across individual micromass cultures upon addition of WNT3A, Supplementary Fig 5 shows analysis 

422

of individual replicates across experiments, compared to GM. Note that there is increased variability even at the lowest concentration of $50 \mathrm{ng} / \mathrm{ml}$ even though this level of WNT3A treatment does not significantly reduce the number of nodules formed (Fig 4a). This indicates a difference in the mechanisms of interference with the pattern of chondrogenesis when BMP signaling is blocked (NOG) and canonical Wnt signaling is activated.

\section{Discussion:}

413 Micromass culture is a valuable tool for a variety of studies; from assaying chondrogenic 414 potential and examining the effect of changing the molecular or mechanical environment on 415 chondrogenic differentiation, to computational modelling of the pattern of nodules that form 416 spontaneously within the culture (Bobick et al., 2014; Christley et al., 2007; Francis-West et 417 al., 1999; Klumpers et al., 2015; Miura et al., 2000; Norrie et al., 2014; Raspopovic et al., 2014; 418 Saha et al., 2017). The regular pattern of chondrogenic nodules that typically forms when 419 primary embryonic limb bud cells are placed under micromass culture conditions is the result 420 of whole culture dynamics (rather than pre-determination), where the foci of differentiating 421 cells self-organise within the culture environment (Raspopovic et al., 2014) and where a Turing like reaction-diffusion mechanism has been proposed to underlie pattern generation (for example Christley et al., 2007). Chondrogenesis in micromass culture has been measured quantitatively using qRT-PCR analysis of marker genes, with rapid increase in both chondrogenic markers (e.g. Sox 9 and Col2a1) and markers of hypertrophy (e.g. Ihh and Col10a1)(Saha et al., 2017), while the pattern of nodule formation is often described more qualitatively with limited use of quantitation. Here we present a novel approach that systematically captures characteristic aspects of the emerging patterns quantitatively. We use the analysis to profile the generation of discrete chondrogenic nodules over time. We show rapid formation of cellular condensations by $24 \mathrm{hrs}$ in culture and, by tracing the elaboration of the pattern in live cultures, that these condensations become the Alcian Blue positive cartilage nodules detectable by $48 \mathrm{hrs}$. We show that nodules form at regular distances from each other, grow in size becoming elongated and rougher, with the edges of adjacent nodules coming closer together over time. In parallel, monitoring the expression of marker genes showed profiles consistent with previous studies (Saha et al., 2017), while we noted high levels of variation between experiments. We further analysed the effect of changing the signaling environment on the pattern, with addition of pro-chondrogenic (BMP2) and anti-chondrogenic (WNT3A and NOG) factors. By titrating the concentration of added factors and comparing the effect of Wnt to the BMP antagonist Noggin, we demonstrate how pattern analysis can be used 
to explore the mechanism(s) involved in pattern generation. We show that although Wnt and Noggin addition both decrease chondrogenesis and nodule number, the effects on the pattern are distinct, indicating that they affect the signaling balance in different ways.

The ability of limb bud cells to spontaneously differentiate, forming regularly spaced nodules reflects an intrinsic capacity to self-organise into this relatively simple pattern and allows for the examination of the basis of such pattern generation. Analysis of the spatial pattern quantitatively is therefore of particular value. The methodology developed here was inspired by large-scale ecological data analysis that quantifies and classifies geographical areas and compares the spacing and proximity of specific parameters using Geographical Information Systems (GIS). We adapted ArcGIS software that is commonly used as a tool to identify spatiotemporal patterns, including viral infections, disease outbreaks and spread (Cunze et al., 2018; Nyakarahuka et al., 2017; Olanrewaju and Adepoju, 2017), the ecological stability of plant species (Miao et al., 2016) and analysis of vacant land for reuse (Newman et al., 2017). In applying this methodology, we treated images of whole cultures (landscapes) as 'maps' and nodules as 'islands' to analyse the spatial relationships amongst them. Previous quantitative analysis of micromass cultures have counted nodules (Bhat et al., 2019; Duke et al., 1998; Saha et al., 2017), measured total chondrogenic area and nodule size (Butterfield et al., 2017), and distances between nodules by utilising binary image processing to quantify periodicity in chondrogenic patterns (Christley et al., 2007; Mello and Tuan, 1999; Miura et al., 2000). Similarly, we use binarisation of images to segregate chondrogenic and non-chondrogenic areas, however in contrast to our approach, previous studies utilised a skeletonisation function to identify the centre line of each chondrogenic area (described in Miura et al., 2000). Thus, while focusing on the average interval between nodules; parameters such as nodule size, shape, and nodule to nodule edge distances were not integrated. A benefit of our approach using ArcGIS, is that it examines multiple parameters including both nodule centres and edges and thus allows a richer examination of the relationship between nodules. For example, it revealed that while addition of increasing concentrations of BMP2 leads to nodules with centre points progressively closer together (Fig $4 \mathrm{c}$ ), the proximity of nodule edges only significantly changed at the highest level of BMP 2 treatment (Fig 4d). The benefit of this approach therefore is that it captures greater detail in monitoring patterns over time and under different treatments; it enables an experimental approach to quantify biological spatial patterns generated in simple culture, results from which can be used in computational models to investigate more specific hypotheses regarding self-organisation and pattern formation in vivo.

The effects of BMP and Wnt signaling are of prime interest for this study because of their 
importance in the spatially appropriate control of cartilage differentiation at forming joints and digit patterning (Raspopovic et al., 2014). In a model proposed by Ray et al. (Ray et al., 2015), adjacent territories of BMP and Wnt signaling underlie the spatial distinction between transient cartilage that acts as a template for bone formation and stable cartilage formation at the joint. We have previously shown that when mechanical stimulation from movement is reduced in both chick and mouse models, the BMP active territory within the skeletal rudiment expands across the joint zone, accompanied by joint line reduction and rudiment fusions (Rolfe et al., 2018; Singh et al., 2018). It is well established that BMP signaling enhances chondrogenesis in culture (Brady et al., 2014; Carlberg et al., 2001; Mendes et al., 2016; Saha et al., 2017) and, in vivo, overexpression of Bmp2 in the embryonic limb has been shown to induce formation of cartilage at the expense of articular cartilage (Duprez et al., 1996). The findings in the present study of increased chondrogenesis upon addition of BMP2 is therefore of no surprise. The importance of BMP signaling for nodule formation within the culture is further reinforced by the anti-chondrogenic effect of addition of the BMP antagonist Noggin. Although the influence of Wnt signaling on skeletogenesis is challenging to unravel, with the potential involvement of multiple Wnt ligands, Frizzled receptors and pathway modulators (Summerhurst et al., 2008), it has been shown that Wnt signaling activity is suppressed during initial chondrogenic differentiation, but is simultaneously active at the joint interzone, where cartilage formation is inhibited (Day et al., 2005; Guo et al., 2004; Rolfe et al., 2018; Singh et al., 2018; Spater et al., 2006). Also, embryonic misexpression of canonical Wnt pathway ligands or overexpression of $\beta$-catenin has been shown to accelerate chondrocyte hypertrophy, induce gaps in cartilaginous rudiments and upregulate joint specific genes, suggestive of the formation of ectopic joints (Hartmann and Tabin, 2000, 2001; Rolfe et al., 2018). This is aligned with the finding here that addition of the canonical Wnt ligand, WNT3A resulted in an overall reduction in expression of chondrogenic genes and a widespread reduction of chondrogenic nodules. Rudnicki and Brown (1997) also previously showed that expression of Wnt1 or Wnt7a using retroviral infection abolished nodule formation (Rudnicki and Brown, 1997). The more surprising and illuminating finding here is that addition of WNT3A not only reduced the number of nodules but also abolished regular distancing between nodules.

A Turing mechanism involving interplay between BMP and Wnt signaling and Sox9 expression has been proposed to regulate the repeated pattern of digit formation in the distal limb bud (Raspopovic et al., 2014). A key feature of Turing mechanisms is their ability to selfregulate or modulate output patterns based on integration of signals (reviewed in Kondo and Miura, 2010). The regular spacing of nodule formation events in micromass cultures recalls 
the spacing of digits in developing hand and footplates, and, is also speculated to be the result of such a Turing mechanism, with different parameters (Christley et al., 2007; Miura et al., 2000; Miura and Shiota, 2000a; Raspopovic et al., 2014). The alteration and titration of the signaling environment in this study indicates that adjusting the signaling balance systemically can impact the regular spacing of nodule formation which could be a result of adjusting the parameters in such a Turing mechanism. A robust method to quantitatively describe and assess nodule formation in micromass culture, as presented here, therefore represents a way to inform testing of proposed Turing mechanisms in vitro, to elucidate the parameters involved in the phenomenal ability of these cells to self organise spatially. While it is unclear whether the mechanisms underpinning the periodic patterns in micromass culture and in digit formation in vivo are the same, our experimental results provide supportive evidence for relationships between the patterns.

Titration of the concentration of added factors to the cultures was valuable in revealing more detail of the effects on the chondrogenic pattern. In all cases, as the concentration of added BMP2, WNT3A and NOG was reduced, the patterns became more similar to that observed in Growth Medium (GM) (Fig 4a), however the effect of increasing concentration of NOG was linear, whereas for BMP2 and WNT3A, lower augmentation levels had proportionally less effect on the pattern. Since Noggin is known to bind BMPs with high affinity (especially 2, 4 and 7) (reviewed in Krause et al., 2011; Zimmerman et al., 1996), this response aligns with a mechanism of sequestration of active BMP under GM culture conditions. It is notable that while increase in the concentration of BMP2 and NOG in the culture media had clear pro- and anti-chondrogenic effects, a pattern of nodules still emerges, showing that changing the systemic concentration within these ranges does not abolish the ability to generate a selforganised pattern. Under the proposed Turning mechanism, this suggests that it does prevent the establishment of reaction-diffusion dynamics. By contrast WNT3A systemic augmentation at $100 \mathrm{ng}$ and $200 \mathrm{ng} / \mathrm{ml}$ abolishes the regular pattern as well as suppressing chondrogenesis. It is interesting to note that previous modelling predicted that if exposure to the activator (BMP in this scenario) is too short, the nodule size would be reduced and the pattern irregular (Christley et al., 2007). The distinct profile of effects of the two anti-chondrogenic factors, WNT3A and NOG, favours a more indirect interplay between Wnt and BMP with increase in the Wnt concentration disrupting the spatial pattern rather than reducing the frequency of differentiation foci (Fig 4c, d, e). Indirect interaction Between Wnt and BMP signaling is a feature of the reaction-diffusion model proposed by Raspopovic et al (Marcon et al., 2016; Raspopovic et al., 2014). 
542 It is interesting to compare the micromass pattern produced by limb bud cells derived at different developmental time points grown in GM (Supplementary Fig 2) to the effects of signaling molecule addition at E11.5. While addition of BMP2 led to larger nodules covering more area, reminiscent of cultures prepared from the earlier stage, E10.5 limb buds, addition of NOG and WNT3A led to formation of smaller nodules covering less area, reminiscent of cultures produced at the later timepoint of E12.5 (Supplementary Table 1 a; Supplementary Fig 2). It would be interesting to investigate the level of BMP and Wnt signaling active within the limb bud across E10.5- E12.5. One indirect means of assessing the level of canonical Wnt signaling would be measuring the activity of read-out genes within the limb buds of canonical Wnt reporter mice (for example: Ferrer-Vaquer et al., 2010; Maretto et al., 2003).

While the micromass culture system has to date been valuable in assessing chondrogenesis, this study reveals greater potential for further insight if the pattern of chondrogenic output is analysed more comprehensively. Chondrogenic output is often measured by quantitative assessment of marker gene expression and while the use of qRT-PCR in this respect is valuable, we found high levels of variation between independent micromass experiments (Supplementary Fig 1 and (Saha et al., 2017)). The marker gene expression profile revealed here was in line with previous studies and reflected the chondorogenic output revealed by analysis of the pattern of nodules formed, however nodule pattern analysis gave a more consistent quantitative measure. Micromass assays can be used to assess a wide range of soluble and physical cues, enabling well-controlled investigation of the role of these varied cues in guiding tissue patterning and boundary formation in skeletal development. For example, the adjustment of substrate stiffness or other aspects of the physical environment, of oxygen tension (recapitulation of embryonic levels), or cytoskeletal mechanics, as well as further exploration of signaling molecules. The effect of creating local gradients of signaling molecules would be of particular interest especially in light of the debate about the relative importance of spatially graded molecular cues and self-organising mechanisms, and the interplay between them (Green and Sharpe, 2015). To date micromass culture has been used to investigate cellular response to the mechanical environment (Juhasz et al., 2014; Onodera et al., 2005; Saha et al., 2017), but combining this with manipulation of the signaling environment holds great potential for furthering our understand of how skeletal patterning is established and tissues organised with wide significance to the fields of synthetic biology and regenerative medicine.

\section{Conclusion:}

This study provides a description of the geometric self-organisation of chondrogenic 
condensations formed from embryonic limb bud cells in micromass culture. It utilises a novel geographical approach in the examination of whole culture patterns of chondrogenesis over time and the impact of molecular manipulation on chondrogenic pattern formation. This analysis captures more quantitative information about nodule to nodule spatial relationships than previous approaches. Future application of the method, and the data it generates, could inform computational models to investigate more specific hypotheses regarding selforganisation mechanisms and pattern formation in vivo.

\section{Acknowledgements:}

All animal work was subject to ethical approval within Trinity College and carried out under licence (to RR and CS) by the Health Products Regulatory Authority. Thanks to Dermot McMurrough with assistance from Jesko Zimmerman at Trinity College for writing the code to calculate proximity data nearest neighbour distances (NearestTable.py). Thanks to Prof Celia Holland for advice on statistical analysis.

Author's contributions:

Conception and design: RR., CS. and PM. Acquisition of data: RR., and CS. Analysis and interpretation: all authors. Writing and revising the article: all authors. Final approval for publication: all authors.

\section{Conflict of Interest : We declare no conflict of interests}

Funding: This work was supported by Trinity College (RR and PM) and an Irish Research Council Postgraduate Studentship (CS).

Ethical Approval: Mice were housed and bred at the Comparative Medicine Unit under accreditation from the Health Products Regulatory Agency (HPRA), Ireland. All animal work was carried out under personal licence from the HPRA. Procedures are approved by the Trinity College Research Ethics Committee where required (all procedures here are covered by licence and no additional ethical approval was required).

608 Ahrens, P.B., Solursh, M., Reiter, R.S., 1977. Stage-related capacity for limb chondrogenesis 609 in cell culture. Dev Biol 60, 69-82. 
Bhat, R., Glimm, T., Linde-Medina, M., Cui, C., Newman, S.A., 2019. Synchronization of

Hes1 oscillations coordinates and refines condensation formation and patterning of the avian limb skeleton. Mech Dev 156, 41-54.

Bobick, B.E., Alexander, P.G., Tuan, R.S., 2014. High efficiency transfection of embryonic limb mesenchyme with plasmid DNA using square wave pulse electroporation and sucrose buffer. BioTechniques 56, 85-89.

Bobick, B.E., Chen, F.H., Le, A.M., Tuan, R.S., 2009. Regulation of the chondrogenic phenotype in culture. Birth Defects Res C Embryo Today 87, 351-371.

Brady, K., Dickinson, S.C., Guillot, P.V., Polak, J., Blom, A.W., Kafienah, W., Hollander, A.P., 2014. Human fetal and adult bone marrow-derived mesenchymal stem cells use different signaling pathways for the initiation of chondrogenesis. Stem Cells Dev 23, 541554.

Butterfield, N.C., Qian, C., Logan, M.P.O., 2017. Pitx1 determines characteristic hindlimb morphologies in cartilage micromass culture. PLoS One 12, e0180453.

Carlberg, A.L., Pucci, B., Rallapalli, R., Tuan, R.S., Hall, D.J., 2001. Efficient chondrogenic differentiation of mesenchymal cells in micromass culture by retroviral gene transfer of BMP-2. Differentiation 67, 128-138.

Christley, S., Alber, M.S., Newman, S.A., 2007. Patterns of mesenchymal condensation in a multiscale, discrete stochastic model. PLoS computational biology 3, e76.

Cunze, S., Kochmann, J., Kuhn, T., Frank, R., Dorge, D.D., Klimpel, S., 2018. Spatial and temporal patterns of human Puumala virus (PUUV) infections in Germany. PeerJ 6, e4255. Day, T.F., Guo, X., Garrett-Beal, L., Yang, Y., 2005. Wnt/beta-catenin signaling in mesenchymal progenitors controls osteoblast and chondrocyte differentiation during vertebrate skeletogenesis. Dev Cell 8, 739-750.

Delgado, I., Torres, M., 2016. Gradients, waves and timers, an overview of limb patterning models. Semin Cell Dev Biol 49, 109-115.

Duke, P.J., Montufar-Solis, D., Hamazaki, T., Sato, A., 1998. Clinorotation reduces number, but not size, of cartilaginous nodules formed in micromass cultures of mouse limbbud cells. Advances in space research : the official journal of the Committee on Space Research (COSPAR) 21, 1065-1072.

Duprez, D., Bell, E.J., Richardson, M.K., Archer, C.W., Wolpert, L., Brickell, P.M., FrancisWest, P.H., 1996. Overexpression of BMP-2 and BMP-4 alters the size and shape of developing skeletal elements in the chick limb. Mech Dev 57, 145-157. 
643 Ferrer-Vaquer, A., Piliszek, A., Tian, G., Aho, R.J., Dufort, D., Hadjantonakis, A.K., 2010. A sensitive and bright single-cell resolution live imaging reporter of $\mathrm{Wnt} / \mathrm{B}$-catenin signaling in the mouse. BMC Dev Biol 10, 121.

Francis-West, P.H., Abdelfattah, A., Chen, P., Allen, C., Parish, J., Ladher, R., Allen, S., MacPherson, S., Luyten, F.P., Archer, C.W., 1999. Mechanisms of GDF-5 action during skeletal development. Development 126, 1305-1315.

Glimm, T., Bhat, R., Newman, S.A., 2020. Multiscale modeling of vertebrate limb development. Wiley Interdiscip Rev Syst Biol Med 12, e1485.

Green, J.B., Sharpe, J., 2015. Positional information and reaction-diffusion: two big ideas in developmental biology combine. Development 142, 1203-1211.

Guo, X., Day, T.F., Jiang, X., Garrett-Beal, L., Topol, L., Yang, Y., 2004. Wnt/beta-catenin signaling is sufficient and necessary for synovial joint formation. Genes Dev 18, 2404-2417. Hartmann, C., Tabin, C.J., 2000. Dual roles of Wnt signaling during chondrogenesis in the chicken limb. Development 127, 3141-3159.

Hartmann, C., Tabin, C.J., 2001. Wnt-14 plays a pivotal role in inducing synovial joint formation in the developing appendicular skeleton. Cell 104, 341-351.

Juhasz, T., Matta, C., Somogyi, C., Katona, E., Takacs, R., Soha, R.F., Szabo, I.A., Cserhati, C., Szody, R., Karacsonyi, Z., Bako, E., Gergely, P., Zakany, R., 2014. Mechanical loading stimulates chondrogenesis via the PKA/CREB-Sox9 and PP2A pathways in chicken micromass cultures. Cell Signal 26, 468-482.

Klumpers, D.D., Smit, T.H., Mooney, D.J., 2015. The effect of growth-mimicking continuous strain on the early stages of skeletal development in micromass culture. PLoS One 10, e0124948.

Kondo, S., Miura, T., 2010. Reaction-diffusion model as a framework for understanding biological pattern formation. Science 329, 1616-1620.

Krause, C., Guzman, A., Knaus, P., 2011. Noggin. Int J Biochem Cell Biol 43, 478-481. Long, F., Ornitz, D.M., 2013. Development of the endochondral skeleton. Cold Spring Harb Perspect Biol 5, a008334.

Marcon, L., Diego, X., Sharpe, J., Müller, P., 2016. High-throughput mathematical analysis identifies Turing networks for patterning with equally diffusing signals. eLife 5 . Maretto, S., Cordenonsi, M., Dupont, S., Braghetta, P., Broccoli, V., Hassan, A.B., Volpin, D., Bressan, G.M., Piccolo, S., 2003. Mapping Wnt/beta-catenin signaling during mouse development and in colorectal tumors. Proc Natl Acad Sci U S A 100, 3299-3304. 
Mello, M.A., Tuan, R.S., 1999. High density micromass cultures of embryonic limb bud mesenchymal cells: an in vitro model of endochondral skeletal development. In vitro cellular \& developmental biology. Animal 35, 262-269.

Mello, M.A., Tuan, R.S., 2006. Effects of TGF-beta1 and triiodothyronine on cartilage maturation: in vitro analysis using long-term high-density micromass cultures of chick embryonic limb mesenchymal cells. J Orthop Res 24, 2095-2105.

Mendes, L.F., Tam, W.L., Chai, Y.C., Geris, L., Luyten, F.P., Roberts, S.J., 2016.

Combinatorial Analysis of Growth Factors Reveals the Contribution of Bone Morphogenetic Proteins to Chondrogenic Differentiation of Human Periosteal Cells. Tissue Eng Part C Methods 22, 473-486.

Miao, Q., Yuan, Y.J., Luo, G.M., Wei, C.H., Rao, Y.Q., Gong, Y.H., Zhang, L., Shao, J., Dong, Y.K., 2016. [Study on ecological suitability of Gardenia jasminoides based on ArcGIS and Maxent model]. Zhongguo Zhong yao za zhi $=$ Zhongguo zhongyao zazhi $=$ China journal of Chinese materia medica 41, 3181-3185.

Miura, T., Komori, M., Shiota, K., 2000. A novel method for analysis of the periodicity of chondrogenic patterns in limb bud cell culture: correlation of in vitro pattern formation with theoretical models. Anat Embryol (Berl) 201, 419-428.

Miura, T., Shiota, K., 2000a. Extracellular matrix environment influences chondrogenic pattern formation in limb bud micromass culture: experimental verification of theoretical models. Anat Rec 258, 100-107.

Miura, T., Shiota, K., 2000b. TGFbeta2 acts as an "activator" molecule in reaction-diffusion model and is involved in cell sorting phenomenon in mouse limb micromass culture. Dev Dyn 217, 241-249.

Newman, G.D., Smith, A.L., Brody, S.D., 2017. Repurposing Vacant Land through Landscape Connectivity. Landscape journal 36, 37-57.

Norrie, J.L., Lewandowski, J.P., Bouldin, C.M., Amarnath, S., Li, Q., Vokes, M.S., Ehrlich, L.I.R., Harfe, B.D., Vokes, S.A., 2014. Dynamics of BMP signaling in limb bud mesenchyme and polydactyly. Dev Biol 393, 270-281.

Nyakarahuka, L., Ayebare, S., Mosomtai, G., Kankya, C., Lutwama, J., Mwiine, F.N., Skjerve, E., 2017. Ecological Niche Modeling for Filoviruses: A Risk Map for Ebola and Marburg Virus Disease Outbreaks in Uganda. PLoS currents 9.

Olanrewaju, O.E., Adepoju, K.A., 2017. Geospatial Assessment of Cholera in a Rapidly Urbanizing Environment. Journal of environmental and public health 2017, 6847376. 
Onodera, K., Takahashi, I., Sasano, Y., Bae, J.W., Mitani, H., Kagayama, M., Mitani, H., 2005. Stepwise mechanical stretching inhibits chondrogenesis through cell-matrix adhesion mediated by integrins in embryonic rat limb-bud mesenchymal cells. Eur J Cell Biol 84, 4558.

713 Raspopovic, J., Marcon, L., Russo, L., Sharpe, J., 2014. Modeling digits. Digit patterning is 714 controlled by a Bmp-Sox9-Wnt Turing network modulated by morphogen gradients. Science $715345,566-570$.

Ray, A., Singh, P.N., Sohaskey, M.L., Harland, R.M., Bandyopadhyay, A., 2015. Precise

719 Rolfe, R.A., Shea, C.A., Singh, P.N.P., Bandyopadhyay, A., Murphy, P., 2018. Investigating 720 the mechanistic basis of biomechanical input controlling skeletal development: exploring the 721 interplay with Wnt signalling at the joint. Philos Trans R Soc Lond B Biol Sci 373.

722 Rudnicki, J.A., Brown, A.M., 1997. Inhibition of chondrogenesis by Wnt gene expression in 723 vivo and in vitro. Dev Biol 185, 104-118.

724 Saha, A., Rolfe, R., Carroll, S., Kelly, D.J., Murphy, P., 2017. Chondrogenesis of embryonic 725 limb bud cells in micromass culture progresses rapidly to hypertrophy and is modulated by 726 hydrostatic pressure. Cell Tissue Res 368, 47-59.

727 Seemann, P., Schwappacher, R., Kjaer, K.W., Krakow, D., Lehmann, K., Dawson, K., 728 Stricker, S., Pohl, J., Ploger, F., Staub, E., Nickel, J., Sebald, W., Knaus, P., Mundlos, S., 729 2005. Activating and deactivating mutations in the receptor interaction site of GDF5 cause 730 symphalangism or brachydactyly type A2. J Clin Invest 115, 2373-2381.

731 Singh, P.N.P., Shea, C., Sonker, S.K., Rolfe, R., Ray, A., Kumar, S., Gupta, P., Murphy, P., 732 Bandyopadhyay, A., 2018. Precise spatial restriction of BMP signaling in developing joints is 733 perturbed upon loss of embryo movement. Development.

734 Spater, D., Hill, T.P., Gruber, M., Hartmann, C., 2006. Role of canonical Wnt-signalling in 735 joint formation. Eur Cell Mater 12, 71-80.

736 Summerhurst, K., Stark, M., Sharpe, J., Davidson, D., Murphy, P., 2008. 3D representation of 737 Wnt and Frizzled gene expression patterns in the mouse embryo at embryonic day 11.5 738 (Ts19). Gene Expr Patterns 8, 331-348.

739 Takacs, R., Matta, C., Somogyi, C., Juhasz, T., Zakany, R., 2013. Comparative analysis of 740 osteogenic/chondrogenic differentiation potential in primary limb bud-derived and 741 C3H10T1/2 cell line-based mouse micromass cultures. Int J Mol Sci 14, 16141-16167. 
signal noggin binds and inactivates bone morphogenetic protein 4. Cell 86, 599-606.

Tables and figures:

746 Table 1: Custom designed primer sequences used for qRT-PCR expression analysis.

\begin{tabular}{|l|l|l|l|}
\hline Gene & Ref Sequence & Fwd & Rv \\
\hline Col2a1 & NM_0332263.3 & AAGTCACTGAACAACCAGATTGAGA & AAGTGCGAGCAGGGTTCTTG \\
\hline Sox9 & NM_011448.4 & CGGCTCCAGCAAGAACAAG & TGCGCCCACACCATGA \\
\hline Col10a1 & NM_009925.4 & TGCAATCATGGAGCTCACAGA & CAGAGGAGTAGAGGCCGTTTGA \\
\hline Ihh & NM_010544.2 & CCACCTTCAGTGATGTGCTTATTT & CGATGACCTGGAAAGCTCTCA \\
\hline Gapdh & NM_008084 & TGGCCTCCAAGGAGTAAGAAAC & GGGATAGGGCCTCTCTTGCT \\
\hline
\end{tabular}

Figure Legends

Figure 1: Methodological pipeline used to analyse the self-organisation pattern of 
clustering of pinpoints, (a"') clustered pinpoints overlaid on the same culture at $144 \mathrm{hrs}$, Alcian Blue stained and thresholded. (b) Chondrogenic profile of E11.5 limb bud micromass cultures stained with Alcian Blue showing appearance of chondrogenic nodules over time. Quantification of (c) nodule number, (d) \% area covered by nodules, (e) average nodule size $\left(\mathrm{x} 1000 \mu \mathrm{m}^{2}\right)$; shape parameters (f) circularity and (g) solidity; and nodule to nodule spatial relationships (h) nodule spacing $(\mu \mathrm{m})$ and (i) nodule proximity $(\mu \mathrm{m})$. Y-axes are the same for $\mathrm{f} \& \mathrm{~g}$, and $\mathrm{h}$ and $\mathrm{i}$. Data is shown as mean \pm SEM. $\# \mathrm{p} \leq 0.01 * \mathrm{p} \leq 0.05, * * \mathrm{p} \leq 0.001, * * * \mathrm{p} \leq 0.0001$. Scale bar $1 \mathrm{~mm}$

Figure 3: Profile of chondrogenesis in E11.5 mouse limb bud cell micromass cultures is altered following the addition of BMP and Wnt signaling molecules. (a) Micromass cultures in growth media (GM) or (b) in the presence of BMP2 $(100 \mathrm{ng} / \mathrm{ml})$ or (c) WNT3A (200ng/ml) over time, stained with Alcian Blue. Scale bar 1mm. (d - i) Geometric analysis showing (d) nodule number, (e); average nodule size $\left(\mu \mathrm{m}^{2}\right),(\mathrm{f}) \%$ area covered by nodules, $(\mathrm{g})$ circularity and nodule to nodule spatial relationships (h) nodule spacing ( $\mu \mathrm{m})$ and (i) nodule proximity $(\mu \mathrm{m})$. Changes in chondrogenic (Col2al) (j) and hypertrophic $(I h h)(\mathrm{k})$ marker gene expression in micromass culture in growth media (GM) or in the presence of BMP2 $(100 \mathrm{ng} / \mathrm{ml})$ or WNT3A (200ng/ml) over time. Expression level in each sample was normalised to Gapdh and shown relative to expression at 0 hours, set to 1 . Data are mean \pm SEM. Blue boxes: growth media, Orange boxes: +BMP2(100ng/ml) group, Yellow boxes: +WNT3A(200ng/ml) group. Black lines indicate significant difference across groups, yellow lines indicate significant difference within the WNT3A group. $\# \mathrm{p} \leq 0.01 * \mathrm{p} \leq 0.05,{ }^{*} \mathrm{p} \leq 0.001, * * * \mathrm{p} \leq 0.0001$

Figure 4: Titration of chondrogenic signaling molecules adjusts the equilibrium in condensation organisation in micromass culture. (a) Proportion of chondrogenic nodules relative to GM at $144 \mathrm{hrs}$ for WNT3A addition (yellow circles; conc. as indicated on left y axis; 200ng/ml,100ng/ml and 50ng/ml), Noggin (grey circles; conc. as indicated on right y axis, $100 \mathrm{ng} / \mathrm{ml}, 50 \mathrm{ng} / \mathrm{ml}$ and $10 \mathrm{ng} / \mathrm{ml}$ ) and BMP2 (orange circles; conc. as indicated on right y axis, $100 \mathrm{ng} / \mathrm{ml}, 50 \mathrm{ng} / \mathrm{ml}$ and $10 \mathrm{ng} / \mathrm{ml}$ ) (b) \% area covered by nodules and (b') average nodule size $\left(\mathrm{x} 1000 \mu \mathrm{m}^{2}\right)$, (c) nodule spacing $(\mu \mathrm{m})$, (d) nodule proximity $(\mu \mathrm{m})$. Data are mean $\pm \mathrm{SEM}$. Blue boxes: growth media, Orange: BMP2 groups, Grey: NOG, Yellow: WNT3A groups. Concentrations of added BMP2, NOG and WNT3A for all graphs are shown against the $\mathrm{x}$-axis of $d$. The y-axes on the right of $\mathrm{c}$ and $\mathrm{d}$ show an expanded scale needed to represent the data following addition of 200ng/ml WNT3A. (b-d) Black lines indicate significant difference 
806 comparing additive with GM; orange, grey and yellow lines indicate significant difference 807 within the BMP2, NOG and WNT3A treatment groups respectively $* p \leq 0.05, * * p \leq 0.001$, $808 * * * \mathrm{p} \leq 0.0001$. (e-e',') profile plots of selected nodule geometric parameters (showing mean 809 values) as BMP2, WNT3A, and Noggin signaling molecule concentrations increase. Upward 810 trends are shown in red, downward trends in blue. Nodule spacing on addition of WNT3A is 811 not shown but indicated as "irregular", as shown by the large variability in the graphs (4c, d) 812 and analysis of individual cultures (Supplementary Fig 5). 

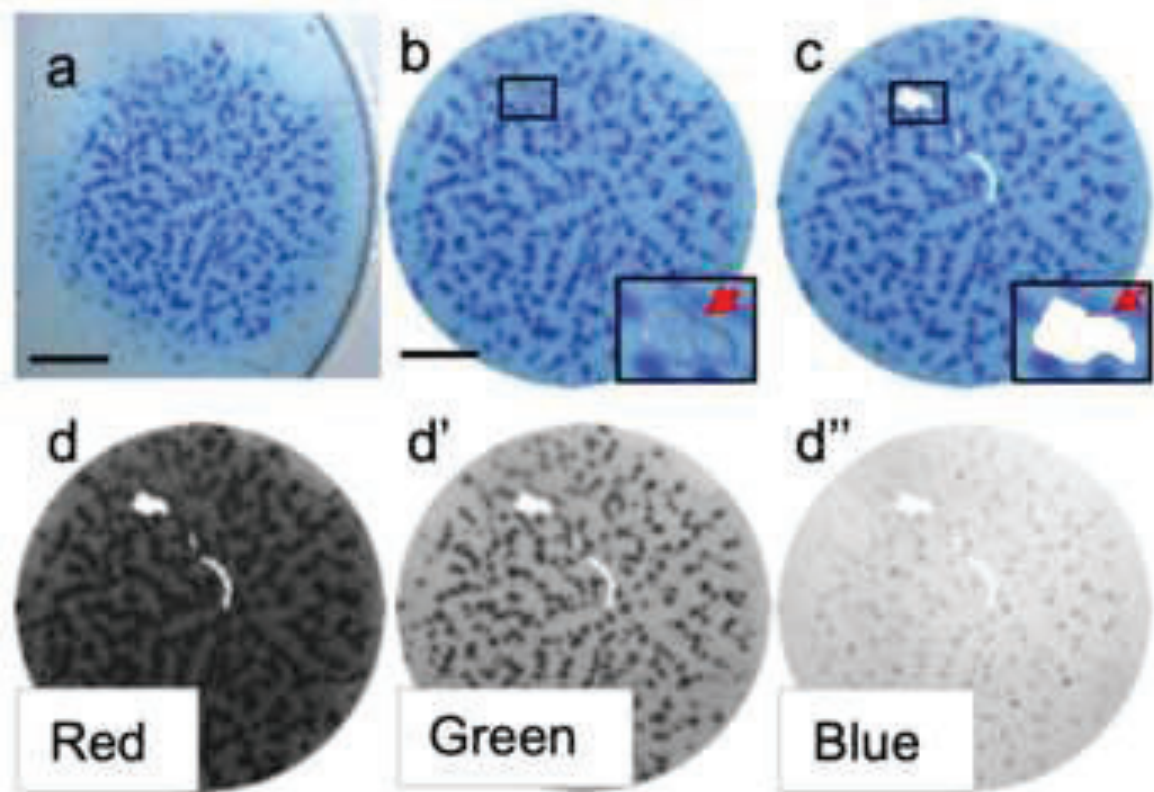

$d^{\prime \prime}$

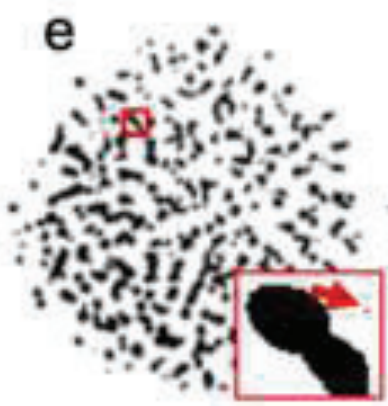

\section{Green}

\section{Blue}

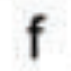

g
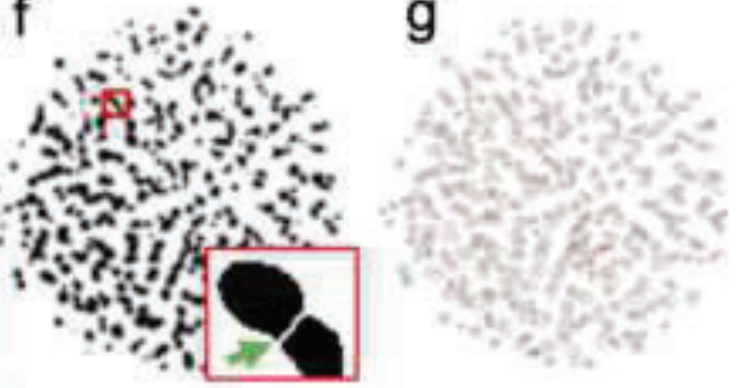

${ }^{\mathrm{h}}$ Circularity
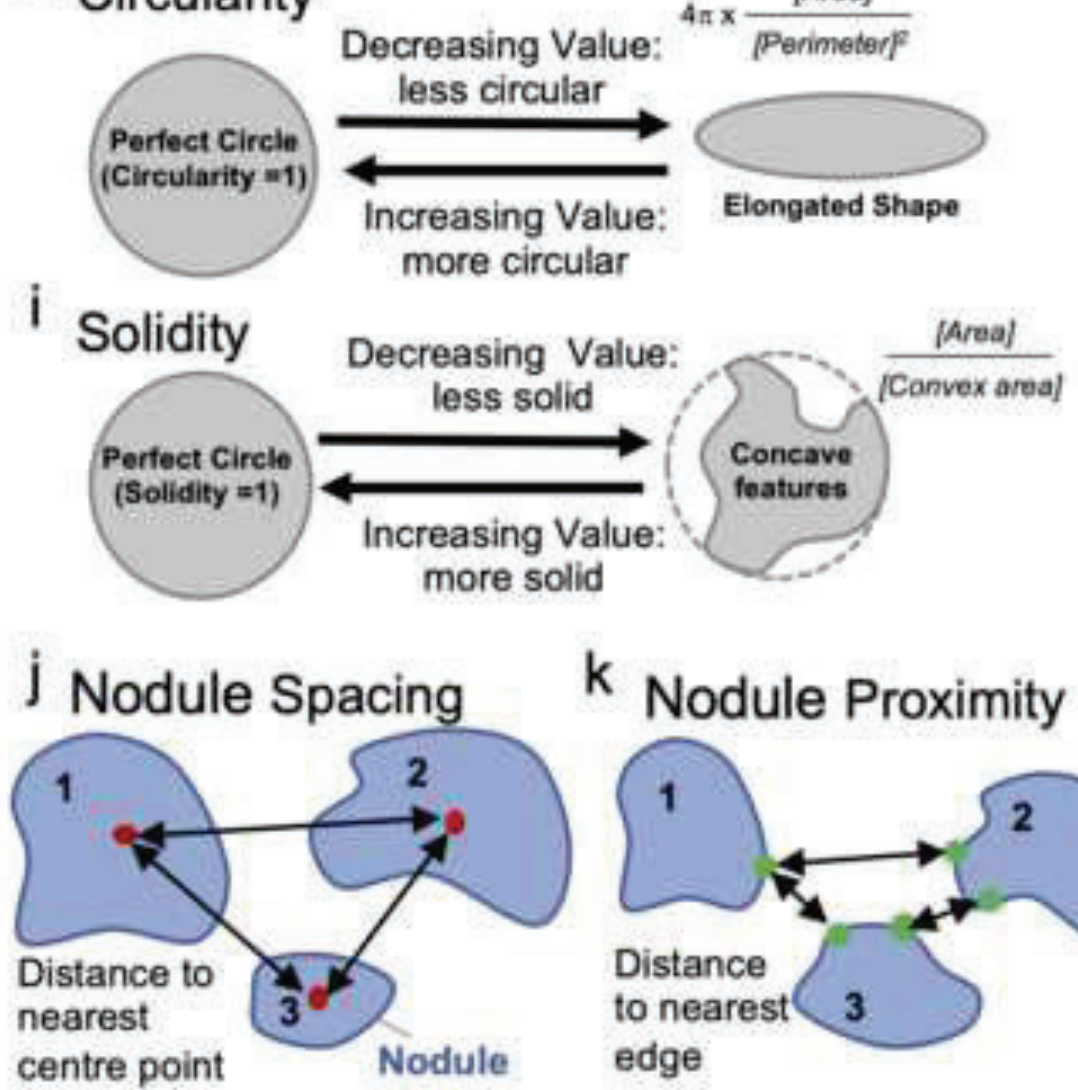

k Nodule Proximity

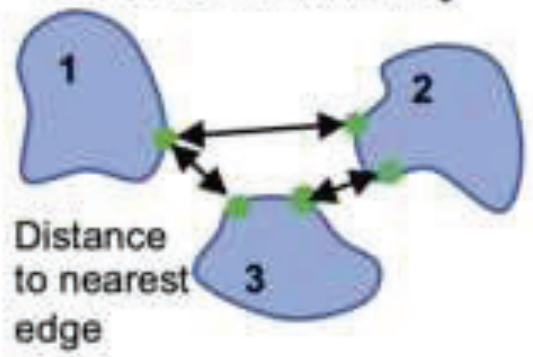




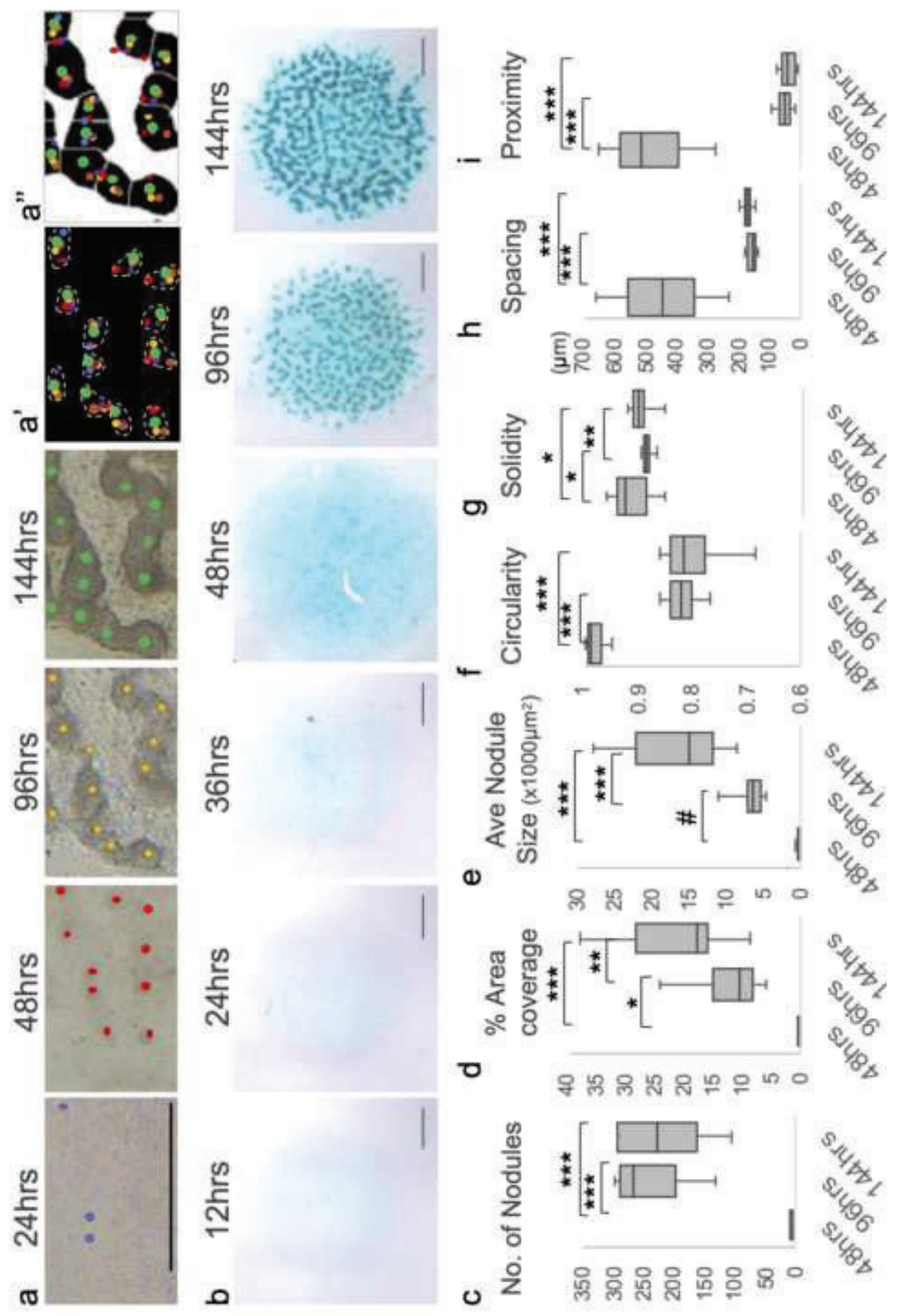




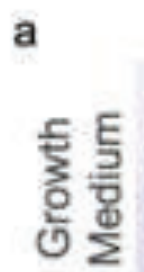

12hrs

24hrs

$36 \mathrm{hrs}$

48hrs

96hrs

144hrs
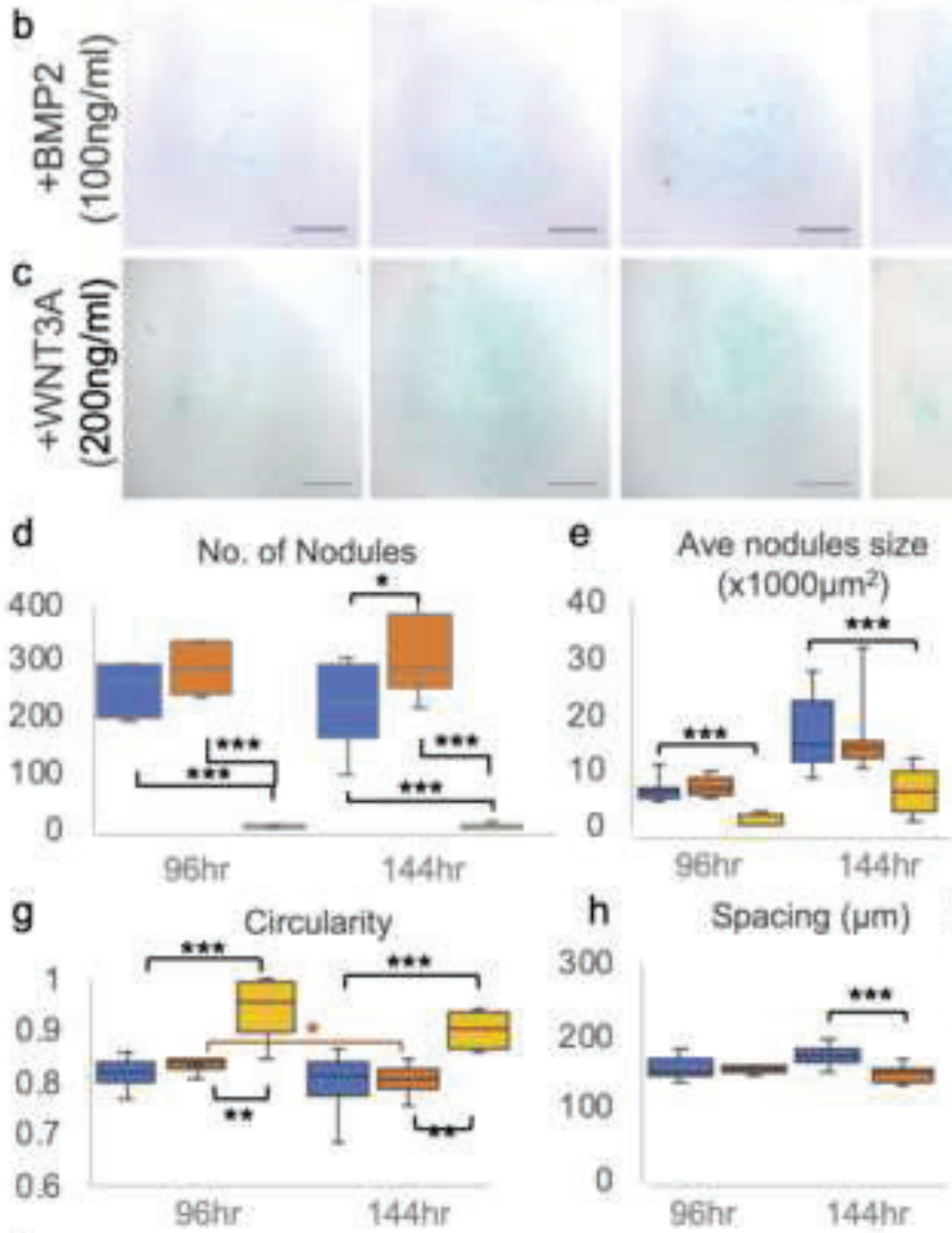

h Spacing $(\mu \mathrm{m}) \quad \mathbf{i}$
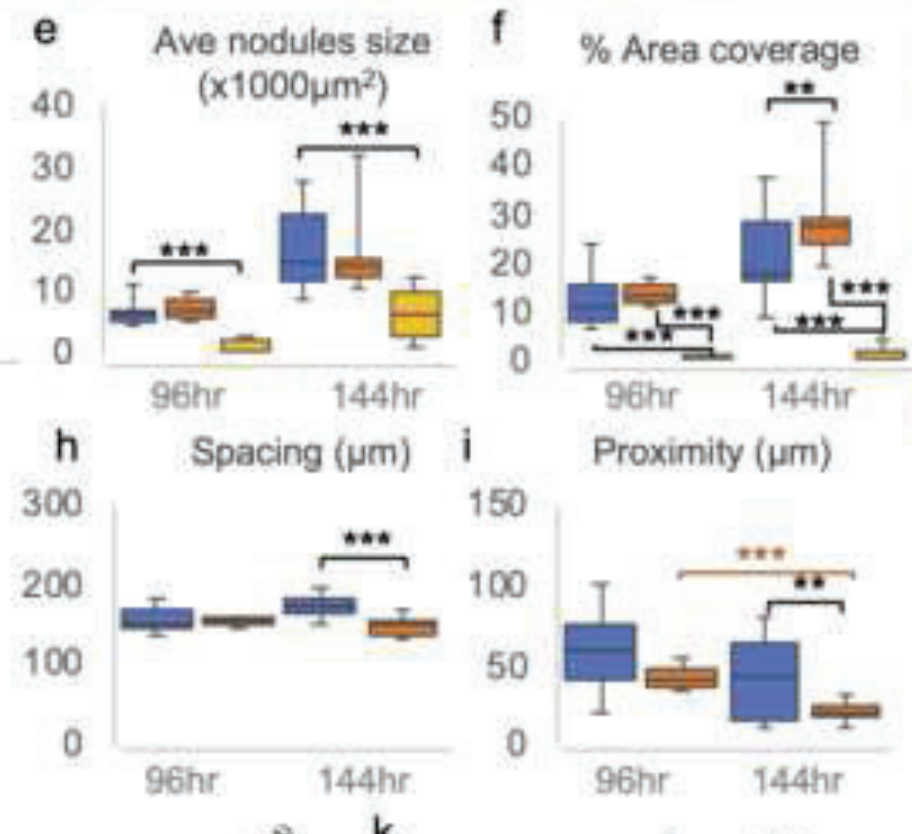

Proximity ( $\mu \mathrm{m})$
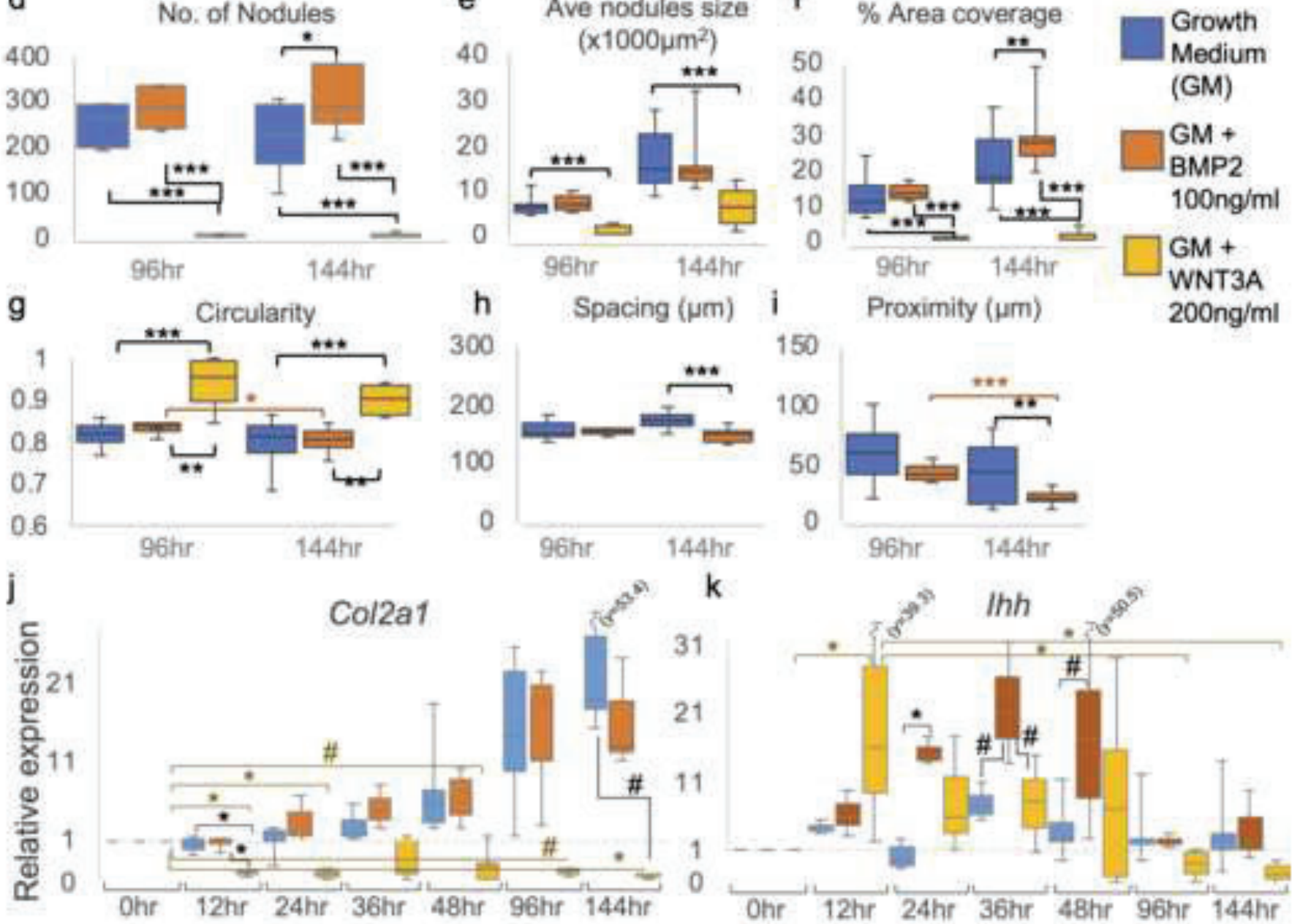

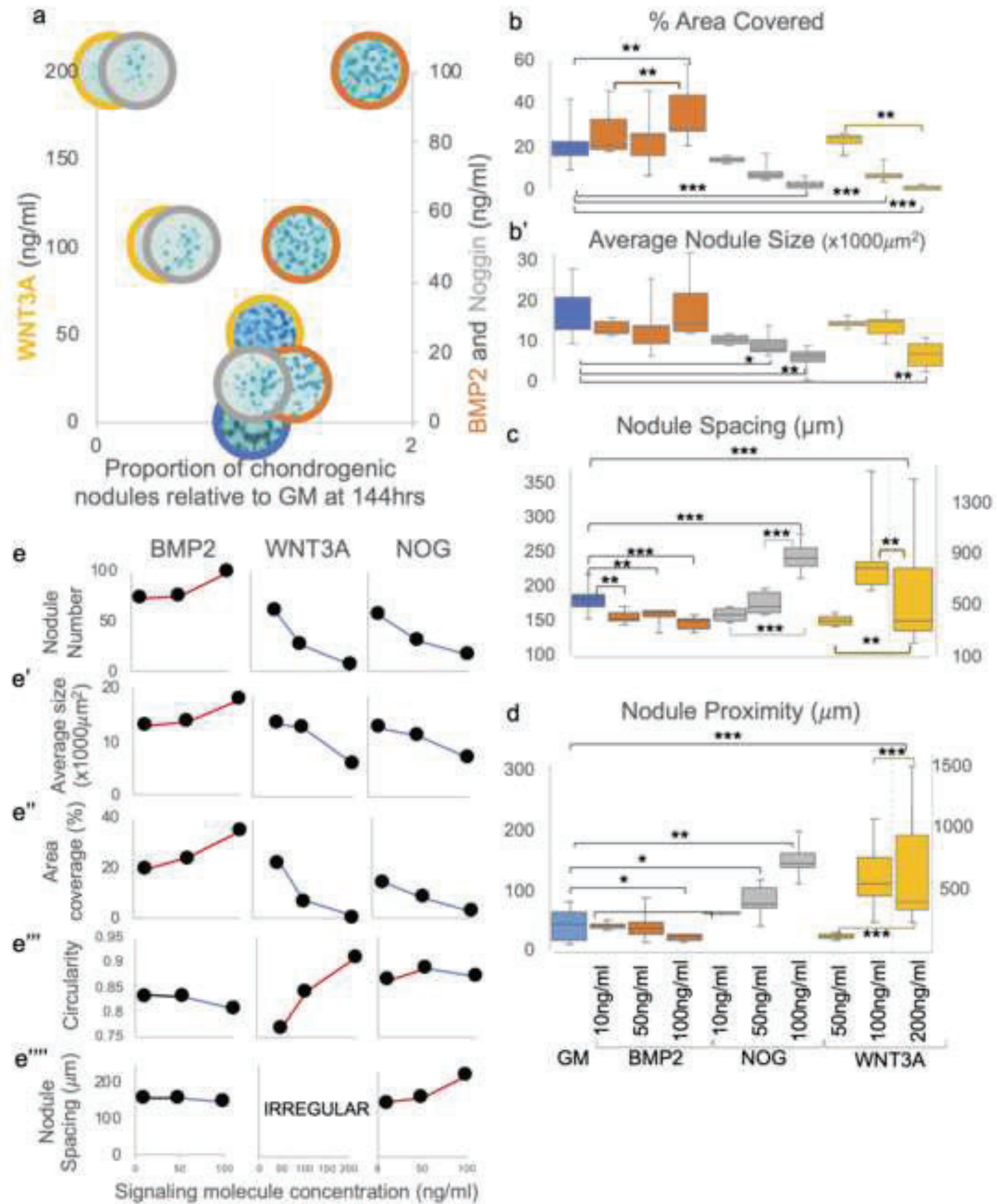
Click here to access/download Supplementary Material Supplementary Material 1.docx 
Click here to access/download Supplementary Material Supplementary Material 2.docx 
Click here to access/download

\section{Supplementary Material}

Supplementary Figures+Tables Revised FINAL.docx 